\title{
APOPTOTIC PROCESSES' RESEARCH ON THE MODEL OF TYPE 2 DIABETES MELLITUS ON OBESITY'S BACKGROUND UNDER THE INFLUENCE OF DENSE BEAN EXTRACT
}

DOI: 10.36740/WLek202008119

\author{
Viktoria A. Rybak, Viktoria V. Korol, Natalia V. Derkach \\ NATIONAL UNIVERSITY OF PHARMACY, KHARKIV, UKRAINE
}

\begin{abstract}
The aim of the study was to observe the influence of dense bean extract on the intensity of apoptotic processes in the liver cells and pancreas of rats on a model of type 2 diabetes mellitus on obesity's background.

Materials and methods: The main method was to model type 2 diabetes mellitus on the background of obesity in organism of mature six-month-old male rats of the Wistar population ( $\mathrm{n}=21$ ), weighing 150-170 $\mathrm{g}$. The modelling was carried out by intraperitoneal low dose administration of streptozotocin $(30 \mathrm{mg} / \mathrm{kg}$, in citrate buffer pH = 4,5) inside after three months period of keeping animals on a combined diet. Apoptosis in DNA samples of liver and pancreas cells was identified in duplicates using electrophoresis in a 1\% agarose gel with using a 1kb DNA SibEnzyme apoptosis marker (from 10,000 to 250 nucleotides).

Results: Only in two of the seven studied DNA samples of the pancreas of a group of rats, treated with a dense bean extract, were observed the traces of necrosis without detectable manifestations of the apoptotic process. It situates at the level of indicators of the animals' intact control group and indicates the distinct effect's presence which includes maintaining pancreas cells survival (in both endocrine and exocrine parts) if imbalance of carbohydrate and lipid metabolism take place in organism.

Conclusion: Dense bean extract showed a more distinct effect than the comparison drug metformin in relation to the risk of premature loss of pancreatic cell function and the development of non-alcoholic fatty liver disease. A dense bean extract is promising for further pharmacological studies, with the aim of creating phytopreparations «Glyphasonorm» tablets and «Glyfasolin» capsules for the correction of type 2 diabetes mellitus and its complications.
\end{abstract}

KEY WORDS: type 2 diabetes mellitus, obesity, metformin, dense bean extract

Wiad Lek. 2020;73(8):1690-1695

\section{INTRODUCTION}

Apoptosis (programmed cell death) is a component of both normal development of the body and pathological health conditions. Specific morphological changes occur in apoptosis: cell shrinkage, chromosome cleavage into nucleosomal fragments, chromatin hypercondensation, intense formation of membrane vesicles without disturbing the integrity of the plasma membrane and formation of apoptotic vesicles surrounded by organelles. During phagocytosis process, neighbor cells absorb apoptotic bodies, which is an immunologically inert process unlike necrosis, which is accompanied by a pronounced inflammatory response. If the activity of apoptosis exceeds the physiological limit, it promotes the development of pathology, characterized by cell loss and impaired organ function $[1,2]$.

Today, apoptosis is intensively studied in many scientific and medical centers due to its basic role in the processes of growth, development, differentiation of tissues, maintenance of tissue homeostasis and functioning of the immune system, as well as cellular alteration, initiation and development of diseases [1]. Apoptosis is one of the fundamental mechanisms that protects organism from cancer and autoimmune diseases. At the same time, this process accompanies myocardial infarction, stroke, septic shock, neurodegenerative diseases, diabetes mellitus (dia- betes) and obesity. It has been proved that excess body mass leads to massive endocrine disorders such are increased activity of the renin-angiotensin-aldosterone system, hyperinsulinemia and insulin resistance, type 2 diabetes, hypercortisolemia, etc. Obesity modulates metabolic effects: increases levels of free fatty acids in serum, production of glycosylation end products, cholesterol level, changes the composition and content of high-, low- and very low-density lipoproteins; activates hemostatic responses (increased erythrocyte sedimentation, plasma fibrinogen concentrations) as well as hematologic responses (erythrocytosis). The identification of specific disorders' mechanisms in the process of programmed cell death regulation allows to clarify the etiology, pathogenesis of this disease, and as a consequence, the possibility to correct the disorders' regulation of apoptosis $[3,4]$.

For example, the direct involvement of the liver in the allogeneic immune response in vivo was determined. The increase in the proportion of functional cytotoxic cells, which are able to attack allogeneic target cells much more actively than spleen lymphocytes, was found in the population of liver lymphocytes. The liver is also considered as a site of migration and death of lymphocytes activated by antigen during the apoptosis mechanism. In any case, the liver directly or indirectly participates in the general host 

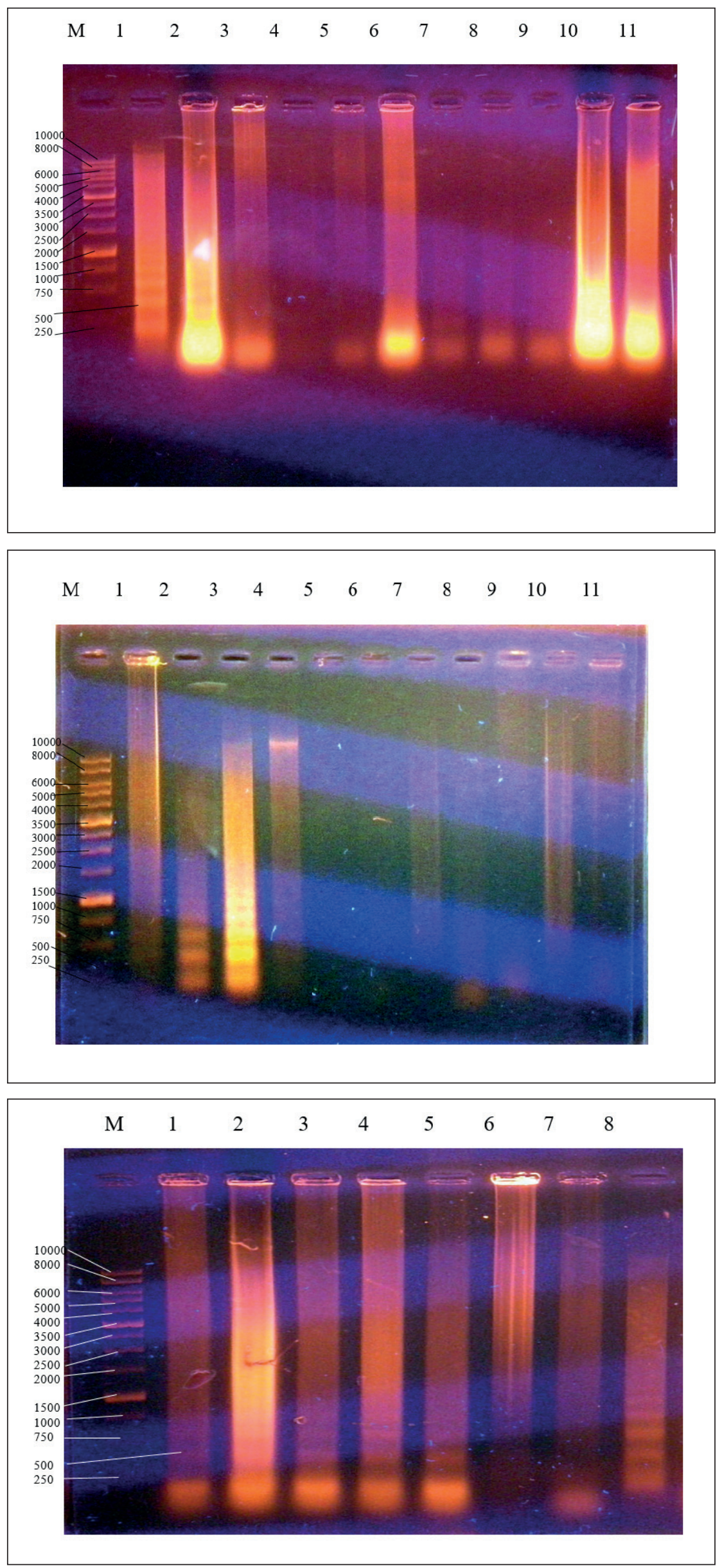

Fig. 1. Photo of gel-electrophoresis DNA "ladders" of rat liver cells on type 2 diabetes model in the background of obesity.

Fig. 2. Photo of of gel-electrophoresis DNA "ladders" of rat liver cells on type 2 diabetes mellitus in the background of obesity.

Fig. 3. Photo of of gel-electrophoresis DNA "ladders" of rat pancreatic DNA on type 2 diabetes mellitus in the background of obesity. 

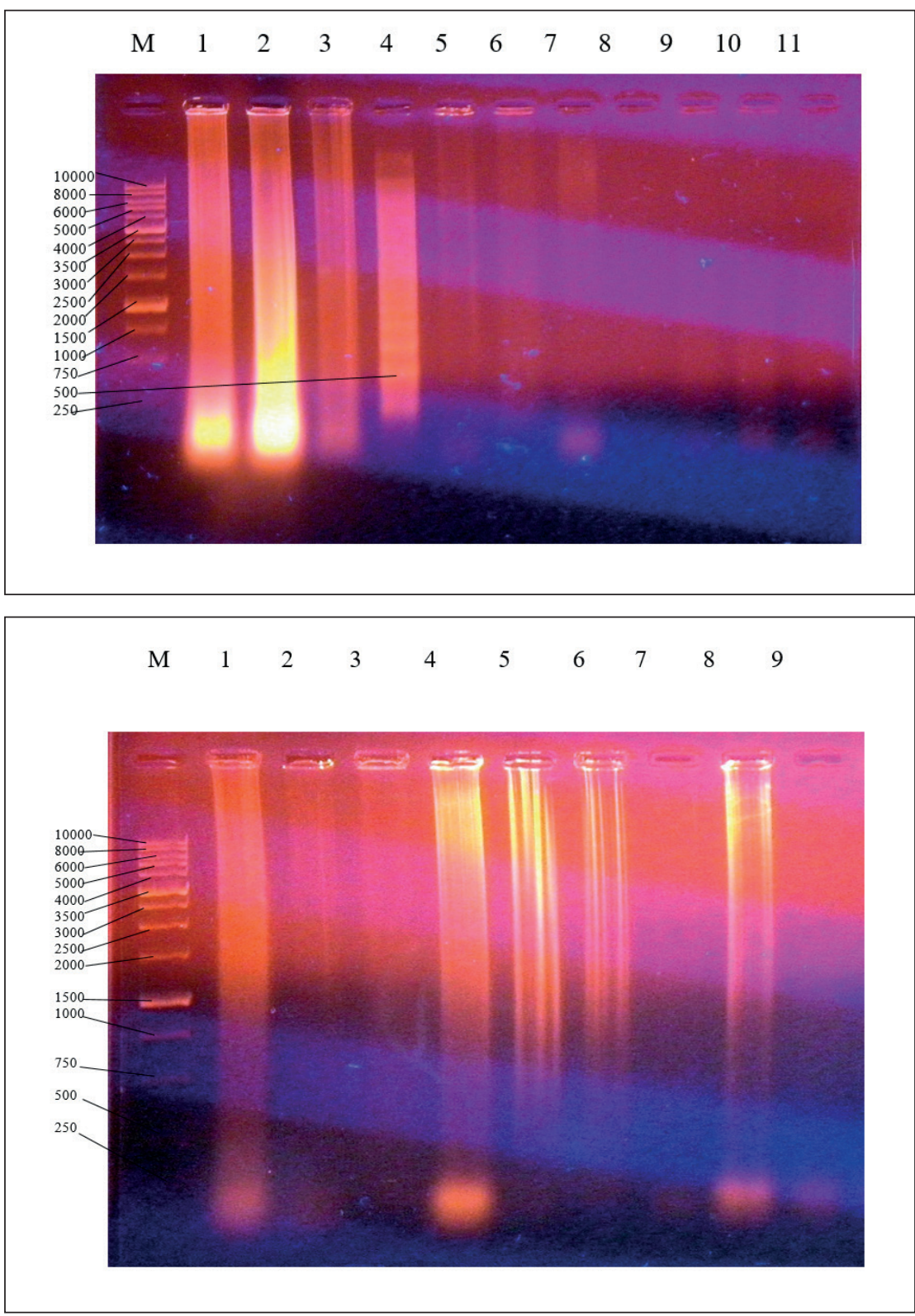

Fig. 4. Photo of of gel-electrophoresis DNA "ladders" of rat pancreatic DNA on type 2 diabetes mellitus in the background of obesity.

Fig. 5. Photo of of gel-electrophoresis DNA "ladders" of rat pancreatic DNA on type 2 diabetes mellitus in the background of obesity. organism's response to the antigenic action. This requires some energy consumption and increased energy production for liver mitochondria involvement in the adaptation process [5].

A large number of agents are capable of inducing apoptosis. In the intact liver, apoptosis is mainly observed in the acinar zone. Apoptosis is detected in the process of various injuries of the liver, but its regulation is not fully determinated. Apoptosis is a fairly rapid phenomenon, for example, in the liver of rats the development of apoptosis takes 3 hours.

T-lymphocytic and lymphocytic cytotoxicity of natural killer cells are often realized through the initiation of apoptosis. It should be noted that, as opposed to necrosis process, during the apoptosis the synthesis of RNA and the corresponding proteins, required to ensure the process of programmed cell death, in hepatocytes increases drastically. In addition, the development of apoptosis in hepatocytes is accompanied by changes in the activity of intracellular signaling systems such as cAMP and protein kinase 3 [1].

The process of apoptosis as one of the mechanisms of $\beta$-cell death in diabetes deserves separate scientific research. The accumulation of knowledge about $\beta$-cell apoptosis in diabetes leads to the development of new strategies that prevent this process of death and, as a consequence, prevent or delay the manifestation of the disease.

One of the most important pro-apoptotic inducers are free radicals, which are significantly increased by chronic hyperglycemia, hyperlipidemia, and mitochondrial dysfunction (mitochondrial dysfunction) under the type 2 diabetes $[4,5]$.

\section{THE AIM}

The aim was to study the effect of thick bean extract on the intensity of apoptotic processes in liver and pancreas cells of rats on the model of type 2 diabetes mellitus on the background of obesity. 


\section{MATERIALS AND METHODS}

Modelling of type 2 diabetes in the organism of six-monthold mature male rats of the Wistar population $(\mathrm{n}=21)$ was performed by administering a low dose of streptozotocin $(30$ $\mathrm{mg} / \mathrm{kg}$ intraperitoneally, on citrate buffer $\mathrm{pH}=4.5$ ) after 90-day combined diet which is a combination of a high fat diet (diet with high content of saturated fat: protein -20.0 $\%$, fat $-60.0 \%$, carbohydrates $-20.0 \%$ of total calories) and excessive carbohydrate intake (free access to fructose solution at a concentration of $200 \mathrm{~g} / \mathrm{l}$ ) [6,7], with a natural changing of lighting, temperature, and humidity standards which are similar to vivarium standards. The control group of animals of the appropriate sex and age $(n=9)$ consumed the standard nutrition (proteins $-15.0 \%$, fats $-5.0 \%$, carbohydrates $-80.0 \%$ of the total calories content), had free access to water and was retained similar conditions.

Thick bean extract was administered orally at a dose of 40 $\mathrm{mg} / \mathrm{kg}$ by probe daily for 30 days, starting from 95 th day of experiment. Metformin (OJSC «Pharmak», Ukraine) was used as a comparison drug in the form of an aqueous suspension with Tween-80 at a dose of $50 \mathrm{mg} / \mathrm{kg}$ body weight according to a similar scheme. The control group received a placebo - 3-5\% aqueous Tween- 80 emulsion according to a similar scheme.

Identification of apoptosis in DNA samples of liver and pancreatic cells was performed in duplicates using a $1 \%$ agarose gel electrophoresis method [8] and adding a $1 \mathrm{~kb}$ DNA apoptosis marker SibEnzyme (10,000 to 250 nucleotide pairs). On electrophoregrams, apoptotic DNA fragmentation is manifested as a «ladder» of DNA fragments of different lengths. Cell necrosis determined the «smeared» nature of the DNA migration zone. The intact DNA glow band was situated near the start.

Experimental studies were conducted according to the requirements of the Commission on Bioethics and the «General Ethical Principles of Experiments on Animals» (Kiev, 2001), which are consistent with the provisions of the «European Convention on the Protection of Vertebrate Animals, are used for experimental and other scientific purposes» (Strasbourg, 1985) [9].

Experimental studies were carried out with the help of the head of Experimental Endocrinology Department of State Institution «Institute of Endocrine Pathology of V. Danilevsky National Academy of Medical Sciences of Ukraine ", medical sciences doctor, professor V. V. Poltorak.

A thick bean extract was chosen for the research, which was obtained from pharmaceutical sciences doctor, professor of Department of Pharmacognosy of the National University of Pharmacy V. M. Kovalev.

\section{RESULTS AND DISCUSSION}

The thick bean extract was obtained from the aboveground part of the common bean (Phaseolus vulgaris L.) and the roots of the Armenian beans, Kharkiv 8 beans and Red local beans varieties. The composition of the thick bean extract includes phenolic compounds represented by flavonols, isoflavones, isoflavonones, isoflavans, pterocarpans, hydroxy acids, coumarins, amino acids.
Pharmacological studies have shown that a thick bean extract at an effective dose of $40 \mathrm{mg} / \mathrm{kg}$ effects on the key pathogenetic links of type 2 diabetes in the background of obesity and its non-specific vascular complications due to the presence of antihyperglycemic, hypolipidemic, antihypertensive, antihypertensive, antiatherogenic and antioxidant properties [10], which creates many conditions for improving the quality and duration of patients' life.

Today, in the conditions of diabetes there is a combined pathology that accelerates the apoptosis of cells in the body.

DNA samples, obtained from the liver of intact control rats, were degraded at the level of 10,000-5,000 nucleotide pairs and above, which is similar in size to the length of DNA in the chromosome loops and can be considered as normal state (Fig. 1, Fig. 2).

The assessment of apoptotic cell death degree of rat liver with type 2 diabetes mellitus in the background of obesity proved that this experimental model, which was accompanied by a significant increase in basal glycemia and the presence of insulin resistance and oxidative stress (data not shown), has caused a remarkable intensivity of hepatocytes' apoptosis.

Thus, in liver samples of rats of the control pathology group, DNA cleavage into fragments of up to 500-250 nucleotide pairs was observed, which indicates a distinct apoptotic process (Fig. 1, Fig. 2), beacuse the size of DNA fragments, that can be fixed on an electrophoregram, reflect the degree of cell death by apoptosis (the smaller the fragments, the more intense the apoptosis). In addition, necrotic tissue damage has been observed, and it can be possibly related to its fatty degeneration and the development of non-alcoholic fatty liver disease [11, 12].

In the liver samples of animals treated with metformin, the intensity of apoptotic DNA breakdown (fragments up to 750 pairs of nucleotides) with the presence of necrosis traces only in single samples, was less observed (Fig. 1).

At the same time, in the group of animals, treated with thick bean extract, electrophoregrams of hepatocyte DNA samples show apoptosis at the limit of the method sensitivity and close to the intact control group detected (Fig. 1, Fig. 2 ), which may indicate the complex recovery of metabolic processes in the liver and coincides with the results of biochemical studies on the presence of low levels of pro- / anti-apoptotic metabolites in both the homogenates of the liver and in the serum of animals - indicators of glycemia, dyslipidemia, lipoperoxidation and antioxidant protection.

It is known that the development of type 2 diabetes is associated with the presence of an inadequate number of functioning pancreatic $\beta$ cells ( $~ 65 \%$ of deficiency in type 2 diabetes manifestations) [13], which is caused by insulin resistance and associated with it glucose- and lipotoxicity $[14,15]$. It is now generally acknowledged that the major mechanism of pancreatic $\beta$-cell mass loss during the diabetes is enhanced apoptosis $[16,17,18]$.

In addition, it should be noted that under the circumstances of obesity, the tension in the exocrine part of the pancreas increases, whose cell necrosis causes a local inflammatory process, and the local increase in the levels of 
proinflammatory cytokines in its turn initiates apoptotic processes in endocrine pancreatic cells $[19,20,21]$.

In the investigated DNA samples from the pancreatic body homogenates of rats of the control pathology group, there were observed many manifestations of apoptotic and necrotic processes, indicating the presence of dysfunction of both exocrine and endocrine part of the gland (Fig. 3, Fig. 4).

In three of the six DNA samples from rat pancreatic homogenates, in the treatment of which metformin was used, the presence of apoptotic DNA breakdown and necrosis traces were observed (Fig. 3, Fig. 4), which characterizes the unsatisfactory exocrine functional state, and may also be associated with some toxic effects of the drug metformin, which is confirmed by the results of electrophoresis of DNA samples of the homogenates of the liver of animals of this group (Fig. 1, Fig. 2).

Only two of the seven pancreatic DNA samples of the rat group, treated with thick bean extract, showed traces of necrosis without fixed manifestations of the apoptotic process, which is situated at the level of the animals from the intact control group (Fig. 4, Fig. 5) and indicates the presence of a strong bean extract with a distinct effect of maintaining the survival of pancreatic cells (both endocrine and exocrine parts) under conditions of imbalance of carbohydrate and lipid metabolism.

Thus, the obtained results obtained indicate the presence in the thick bean extract the properties of preserving the survival of liver cells and pancreas under the conditions of a long-term use in the treatment of animals with experimental type 2 diabetes on the background of obesity.

\section{CONCLUSIONS}

1. Electrophoregrams of DNA samples of liver cells and pancreas of rats with experimental diabetes mellitus type 2 under the conditions of obesity, which received a thick bean extract in the treatment for a month, showed significantly lower manifestations of the apoptotic process than in the samples from the group of rats, treated with the comparison drug Metformin, that may be associated with its significantly higher level of toxicity.

2. Thick bean extract showed a more distinct effect than the Metformin comparison drug, in comparison of reducing the risk of premature loss of pancreatic cell function and the development of non-alcoholic fatty liver disease.

3. Thick bean extract is promising for further pharmacological studies, including the involvement of histomorphological methods of studying its effect on the pancreas, in order to create phytopreparations - "Glyphasonorm» tablets and «Glyphasolin» capsules for correction of type 2 diabetes its complications.

\section{REFERENCES}

1. Khlamanova L.I., Severilova M.D., Tkachenko Yu.V. Morphofunctional features of apoptosis, problems and prospects of apoptosis in modern medicine. Ukrainian Journal of Medicine, Biology and Sports. 2017; 2:185-192.
2. Zamaraev A.V., Kopeina G.S., Prokhorova E.A. et al. Post-translational Modification of Caspases: The Other Side of Apoptosis Regulation. Trends in cell biology. 2017; 27(5):322-339. doi:10.1016/j.tcb.2017.01.003.

3. Ashcheulova T.V. Apoptosis: signaling pathways and significance in cardiometabolic pathology. Kharkov. 2016. $110 \mathrm{p}$.

4. Guimaraes C.A., Linden R. Programmed cell deaths. Apoptosis and alternative deathstyles. Europ. J. Biochem. 2004; 27:1638-1650.

5. Martin S.D., McGee S.L. The role of mitochondria in the aetiology of insulin resistance and type 2 diabetes. Biochim. Biophys. Acta. 2014; 1840, 4:1303-1312.

6. Jurgoński A., Juśkiewicz J., Zduńczyk Z. High-fat diet differentially affects the gut metabolism and blood lipids of rats depending on the type of dietary fat and carbohydrate. Nutrients. 2014; 6, 2:616-626.

7. Damasceno D.C., Netto A.O., lessi I.L. et al. Streptozotocin-induced diabetes models: pathophysiological mechanisms and fetal outcomes. BioMed. Research International. 2014, Article ID 819065. 11 p.

8. Sokolov B.P., Dzhemelinsky V.V. Isolation of high molecular weight eukaryotic DNA using potassium acetate. Molecular genetics, microbiology and virology. 1989; 6:45-46.

9. General ethical principles of animal experiments. Endocrinology. 2003; 8, 1:142-145.

10. RybakV., Derkach N., Korol V. Modern aspects of sugar-reducing effect of thick bean extract based on a type ii diabetes model on the background of obesity. Georgian medical news. 2019; 5(290):144-149.

11. Takamura T., Misu H., Ota T. et al. Fatty liver as a consequence and cause of insulin resistance: Lessons from type 2 diabetic liver. Endocrine Journal. 2012;59, 9:745-763.

12. Dumas M-E., Kinross J., Nicholson J.K. Metabolic phenotyping and systems biology approaches to understanding metabolic syndrome and fatty liver disease. Gastroenterology. 2014; 46:P. 46-62.

13. Saisho Y. $\beta$-cell dysfunction: Its critical role in prevention and management of type 2 diabetes. World J. Diabetes. 2015; 6, 1:109-124.

14. Costes S., Langen R., Gurlo T. et al. $\beta$-Cell failure in type 2 diabetes: a case of asking too much of too few? Diabetes. 2013; 62, 2:327-335.

15. Sharma R.B., Alonso L.C. Lipotoxicity in the pancreatic beta cell: not just survival and function, but proliferation as well? Curr. Diab. Rep. 2014; 14, 6:492.

16. Cernea S., Dobreanu M. Diabetes and beta cell function: from mechanisms to evaluation and clinical implications. Biochem. Med. (Zagreb). 2013;23, 3:266-80.

17. Quan W., Jo E.K., Lee M.S. Role of pancreatic $\beta$-cell death and inflammation in diabetes. Diabetes Obes. Metab. 2013; 15, 3:141-51.

18. Lee S.C., Pervaiz S. Apoptosis in the pathophysiology of diabetes mellitus. Int. J. Biochem. Cell Biol. 2007; 39:497-504.

19. Agrawal N.K., Kant S. Targeting inflammation in diabetes: Newer therapeutic options. World J. Diabetes. 2014;5, 5:697-710.

20. Montane J., Cadavez L., Novials A. Stress and the inflammatory process: a major cause of pancreatic cell death in type 2 diabetes. Diabetes Metab. Syndr. Obes. 2014;7:25-34.

21. Kitamura T. The role of FOX01 in $\beta$-cell failure and type 2 diabetes mellitus. Nat. Rev. Endocrinol. 2013; 9, 10:615-623.

The work is a fragment of the research work of the $\mathrm{Na}$ tional University of Pharmacy, approved by the Ministry of Health of Ukraine: "Pharmacological study of biologically active substances and drugs» (№ state registration 0114U000956). 


\section{ORCID and contributionship:}

Viktoria A. Rybak: 0000-0001-7649-4287 A,E,F

Viktoria V. Korol: 0000-0001-9483-6906 B,D

Natalia V. Derkach: 0000-0002-8827-3534 ${ }^{\mathrm{C}}$

\section{Conflict of interest:}

The Authors declare no conflict of interest.

\section{CORRESPONDING AUTHOR}

Viktoria A. Rybak

National University of Pharmacy

22 Architects str., 61174 Kharkiv, Ukraine

tel: +380501677254

e-mail:viktoriarybak2@gmail.com

Received: 14.10 .2019

Accepted:02.06.2020

A - Work concept and design, B - Data collection and analysis, C - Responsibility for statistical analysis,

$\mathbf{D}$-Writing the article, $\mathbf{E}$-Critical review, $\mathbf{F}$ - Final approval of the article 